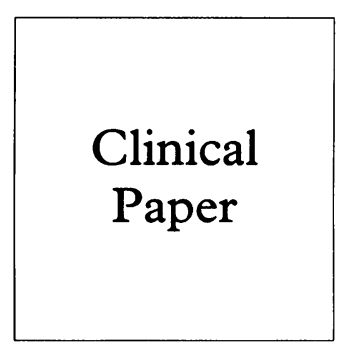

\title{
Identifying cervical infection among pregnant women in Nairobi, Kenya: limitations of risk assessment and symptom-based approaches
}

\author{
T Thomas, S Choudhri, C Kariuki, S Moses
}

Objectives: To examine characteristics of pregnant women associated with cervical infection, and to evaluate the accuracy of symptom-based and risk assessment systems which have been developed for identifying cervical infection in antenatal women.

Methods: Interviews were conducted and physical examinations performed on 291 consecutive antenatal clinic attenders in Nairobi, Kenya. Vaginal, cervical, urine and blood specimens were also obtained for analysis.

Results: The following disease prevalences were observed: candidiasis $26 \cdot 2 \%$; trichomoniasis $19.9 \%$; bacterial vaginosis $20.6 \%$; any vaginal infection $53.8 \%$; chlamydial cervicitis (CT) $8 \cdot 8 \%$; gonococcal cervicitis (GC) $2 \cdot 4 \%$; any cervical infection $10.8 \%$. The only statistically significant association with GC and/or CT cervical infection was the presence of cervical friability (OR = $2 \cdot 1, P=0 \cdot 05)$. There were trends towards associations with the presence of endocervical mucopus $(\mathrm{OR}=2 \cdot 6, \mathrm{P}=0 \cdot 06)$, reporting a new sex partner in the past 3 months $(\mathrm{OR}=2 \cdot 2, \mathrm{P}=$ $0 \cdot 16)$ and reporting that a sex partner had an STD-related symptom (OR $=4 \cdot 4, P=0 \cdot 13)$. There were no associations with other demographic, behavioural or medical characteristics. Risk scores previously developed for detecting GC/CT cervicitis in developing country antenatal populations generally performed poorly.

Conclusions: The prevalences of vaginal and cervical infection observed were extremely high among these "low risk" women. Owing probably to high levels of vaginal infection and to behavioural characteristics of this urban population, factors which elsewhere have been associated with cervical infection were not found to be so in this setting. Further work on symptom-based approaches and risk assessment for STD case detection in pregnant women is required before STD management recommendations can be generalised.

(Genitourin Med 1996;72:334-338)

Keywords: STD case management; antenatal women; risk assessment; developing countries

Department of

Medical Microbiology,

University of Manitoba

T Thomas

S Choudhri

$S$ Moses

Department of

Medical Microbiology,

University of Nairobi

$S$ Choudhri

C Kariuki

Department of

Community Health,

University of Nairobi

and Department of

Community Health

Sciences, University of

Manitoba

S Moses

Address correspondence to:

Dr Stephen Moses,

Dr Stephen Moses,

Department of Medical
Microbiology, University of

Microbiology, University
Manitoba, 730 William

Manitoba, 730 William
Avenue, Winnipeg,

Manitoba, R3E 0W3,

Canada.

Accepted for publication

10 July 1996

\section{Introduction}

The detection and management of sexually transmitted diseases (STDs) in individuals who present to health facilities is an important component of STD control strategies. Early diagnosis and effective treatment reduces an individual's infectivity, and contact with a health provider provides opportunity for advice on the importance of reducing risktaking behaviour and for ensuring that partners are effectively treated. Effective management should be provided at the point of first contact with the health system, and extend throughout all health facilities offering care. ${ }^{1}$ As many women in developing countries routinely attend antenatal, family planning, and maternal and child health clinics, there are potentially important opportunities in these settings to detect and effectively manage STDs. There is therefore a great deal of interest at the present time in attempting to integrate STD-related services into women's health care. $^{2}$

Because of the general lack of adequate laboratory facilities and the difficulties in making accurate specific clinical diagnoses, simplified STD management algorithms, using syndromic approaches, have been developed and implemented in many African countries.
Patients presenting to health facilities are diagnosed and treated on the basis of their presenting symptoms, with some or all of the aetiological possibilities covered in a standardised manner. Guidelines using simple algorithms have been developed by the World Health Organization and others. ${ }^{3}$ To identify individuals with asymptomatic infection and in some situations to improve the accuracy of algorithms for syndromic STD management, there are two additional options. One is to apply risk assessment, in which risk scores are developed based on a combination of characteristics associated with the individual, with these scores used to predict the presence of infection..$^{4-6}$ The second is to include simple "bedside" biological tests to improve diagnostic accuracy, such as the leukocyte esterase dipstick test (LED) to predict gonococcal or chlamydial urethral infection in $\operatorname{men}^{7-9}$ and cervical infection in women. ${ }^{5}$

Unfortunately, little evaluation of the validity of these approaches has been undertaken. Furthermore, as validity will likely vary with different populations, evaluation is required in a variety of settings. As cervical infection is a source of considerable morbidity among women, we examined the potential value of risk assessment and a symptom-based 
approach in detecting cervical infection among a population of antenatal clinic attenders in Nairobi, Kenya.

\section{Methods \\ The study was conducted in June and July 1994 among 291 consecutive women attend- ing an antenatal clinic operated by the Nairobi City Council serving a low socio-economic status population. After obtaining informed consent, demographic and behavioural infor- mation was collected, as well as a medical his- tory. Abdominal and pelvic examinations were then performed. After insertion of an unlubri- cated speculum, the cervix was cleaned using a large cotton swab and examined for the pres- ence of cervical ectopy or ulceration. Vaginal material was obtained from the lateral fornix for wet preparation. A sterile dacron swab was then placed in the endocervix and rotated to obtain a specimen for the leukocyte esterase dipstick test. This specimen was also assessed for mucopurulence and induced bleeding (fri- ability). Subsequent endocervical swabs were obtained for gram stain and culture for Neisseria gonorrhoeae and for Chlamydia tra- chomatis enzyme immunoassay (Syva MicroTrak Chlamydia EIA, Syva, San Jose, USA).}

The endocervical swab for LED testing was placed in a $1.5 \mathrm{ml}$ tube containing 8 drops of normal saline and then agitated. The LE dipstick (Uristix, Miles Canada, Etobicoke, Canada) was then placed in the solution and after two minutes read against the colour standards of the manufacturer. An LED test was also performed on a specimen of first catch urine. After pre-test counselling, blood specimens were obtained for syphilis serology by the rapid plasma reagin test (Macro-Vue RPR Card Tests, Becton Dickinson International, Meylan, France) and HIV serology (Detect HIV 1/2, BioChem ImmunoSystems, Montréal, Canada). Positive RPR tests were confirmed by a Treponema pallidum haemaglutination test (TPHA Test Kits, Biotec Laboratories, Ipswich, UK), and positive HIV serology by a second EIA (Recombigen HIV 1/2, Cambridge Biotech, Galway, Ireland). Women were asked to

Table 1 Prevalence of vaginal and cervical infections, as well as other sexually transmitted diseases, among antenatal clinic attenders $(N=286)$

\begin{tabular}{lcc}
\hline & Number & Percent \\
\hline Vaginal infection: & 75 & \\
Candidiasis & 57 & $26 \cdot 2 \%$ \\
Trichomoniasis & 59 & $19 \cdot 9 \%$ \\
Bacterial vaginosis & 154 & $20 \cdot 6 \%$ \\
Any vaginal infection & 25 & $53 \cdot 8 \%$ \\
Cervical infection: & 7 & $8 \cdot 8 \%$ \\
Chlamydial cervicitis & 31 & $2 \cdot 4 \%$ \\
Gonococcal cervicitis & 9 & $10 \cdot 8 \%$ \\
Any cervical infection & 26 & $3 \cdot 1 \%$ \\
Other STDs: & 3 & $9 \cdot 1 \%$ \\
Active syphilis (positive RPR and TPHA) & 9 & $1 \%$ \\
HIV-1 infection & 107 & $3 \cdot 1 \%$ \\
Genital ulcer & 90 & $37 \cdot 4 \%$ \\
Genital warts & & $31 \cdot 5 \%$ \\
Any STD & & \\
Any treatable STD† & & \\
\hline
\end{tabular}

${ }^{\star}$ Candidiasis and bacterial vaginosis excluded.

†Trichomoniasis, chlamydial cervicitis, gonococcal cervicitis, genital ulcer or active syphilis. return one week later for the results of their tests, and those testing positive were counselled and treated according to the recommended guidelines of the Kenyan Ministry of Health. Five women declined a pelvic examination and were excluded from analysis.

Wet preparations were made up by placing vaginal swabs into $0.2 \mathrm{ml}$ of normal saline and putting a drop of the resulting solution onto a slide for microscopic examination. Trichomonas vaginalis infection (trichomoniasis) was defined as the presence of motile trichomonads on wet preparation. Bacterial vaginosis was defined as the presence of clue cells constituting greater than $20 \%$ of all vaginal epithelial cells in high power fields. ${ }^{11}$ Vaginal $\mathrm{pH}$ was measured and the amine odour test on vaginal fluid placed in $10 \%$ potassium hydroxide solution was also performed, but as these tests did not alter the diagnostic yield based on the presence of clue cells alone, only the clue cell data are reported. Candida albicans infection (candidiasis) was defined as the presence of pseudo-hyphae or budding yeast forms on wet preparation, after adding one drop of potassium hydroxide solution. Cultures for $N$ gonorrhoeae were performed on Thayer-Martin media, and incubated and identified according to standard techniques. Chlamydia EIA, and HIV and syphilis serological tests, were performed according to the manufacturers' instructions.

\section{Results}

The mean age of the study population was 23.6 years (SD 4.8 years), $88.5 \%$ of women were married, $71.0 \%$ reported more than one lifetime sex partner and $7 \cdot 0 \%$ reported a new sex partner within the previous three months. There were $18.5 \%$ of women reporting the symptom of vaginal discharge, $9 \cdot 1 \%$ reporting dysuria, $17 \cdot 1 \%$ vaginal itching and $37 \cdot 4 \%$ lower abdominal pain. Approximately $2 \%$ of the women reported STD-related symptoms in their male sex partner. Only one woman reported receiving money for sex and only one woman reported condom use within the previous three months. The prevalences of vaginal infection, cervical infection, syphilis and HIV-1 infection are shown in table 1 . Over half of the women had at least one type of vaginal infection and many had more than one simultaneously. Almost $11 \%$ of the women had either gonococcal or chlamydial cervicitis, and one woman had a dual cervical infection. Of the 31 women with cervical infection, 17 (55\%) also had at least one vaginal infection. There was no statistical association, however, between the presence of cervical infection and the presence of vaginal infection. Over $3 \%$ of the women were sero-reactive for syphilis and almost $10 \%$ were infected with HIV-1. Small numbers had genital ulcers or genital warts. Overall, $37 \%$ of the women had at least one sexually transmitted disease (candidiasis and bacterial vaginosis excluded), and almost onethird had an STD that was treatable.

Table 2 examines various potential risk factors for their association with cervical infec- 
Table 2 Characteristics of antenatal clinic attenders with and without gonococcal and/or chlamydial cervical infection $(N=286)$

\begin{tabular}{|c|c|c|c|c|}
\hline & $\begin{array}{l}\text { Infected } \\
(N=31)\end{array}$ & $\begin{array}{l}\text { Uninfected } \\
(N=255)\end{array}$ & $\begin{array}{l}\text { Odds } \\
\text { ratio }\end{array}$ & $\begin{array}{l}P \\
\text { value }\end{array}$ \\
\hline \multicolumn{5}{|l|}{ Risk determinants: } \\
\hline Age $<25$ years & $74 \cdot 2 \%$ & $62 \cdot 0 \%$ & $1 \cdot 8$ & $0 \cdot 13^{\star}$ \\
\hline Unmarried status & $9 \cdot 7 \%$ & $11 \cdot 8 \%$ & $0 \cdot 8$ & NS \\
\hline$>1$ sex partner in past 3 months & $9 \cdot 7 \%$ & $6 \cdot 7 \%$ & 1.5 & NS \\
\hline$>1$ lifetime sex partner & $74 \cdot 2 \%$ & $70.6 \%$ & $1 \cdot 2$ & NS \\
\hline New sex partner in past 3 months & $12.9 \%$ & $6 \cdot 3 \%$ & $2 \cdot 2$ & $0 \cdot 16^{\star}$ \\
\hline Partner has STD-related complaint & $6 \cdot 5 \%$ & $1 \cdot 6 \%$ & $\mathbf{4} \cdot \mathbf{4}$ & $0 \cdot 13^{\star}$ \\
\hline No other pregnancy within 5 years & $3 \cdot 2 \%$ & $3 \cdot 5 \%$ & 0.9 & NS \\
\hline Never used condom & $10.0 \%$ & $5 \cdot 1 \%$ & $2 \cdot 1$ & $0 \cdot 23^{\star}$ \\
\hline \multicolumn{5}{|l|}{ Symptoms: } \\
\hline Vaginal discharge & $19 \cdot 4 \%$ & $18 \cdot 4 \%$ & $1 \cdot 0$ & NS \\
\hline Dysuria & $9 \cdot 7 \%$ & $9 \cdot 0 \%$ & $1 \cdot 1$ & NS \\
\hline Vaginal itch & $12 \cdot 9 \%$ & $17 \cdot 6 \%$ & 0.7 & NS \\
\hline Lower abdominal pain & $35 \cdot 5 \%$ & $37 \cdot 6 \%$ & $1 \cdot 0$ & NS \\
\hline Dyspareunia & $22 \cdot 6 \%$ & $18 \cdot 0 \%$ & $1 \cdot 3$ & NS \\
\hline \multicolumn{5}{|l|}{ Signs: } \\
\hline Vaginal discharge & $35 \cdot 5 \%$ & $31 \cdot 8 \%$ & $1 \cdot 2$ & NS \\
\hline Endocervical mucopus & $16 \cdot 1 \%$ & $6 \cdot 3 \%$ & $2 \cdot 6$ & $0.06^{\star}$ \\
\hline Cervical friability & $38 \cdot 7 \%$ & $23.0 \%$ & $2 \cdot 1$ & $0.05^{\star}$ \\
\hline Cervical ectopy & $12 \cdot 9 \%$ & $9 \cdot 5 \%$ & 1.4 & NS \\
\hline Adnexal tenderness & $9 \cdot 7 \%$ & $11 \cdot 0 \%$ & 0.9 & NS \\
\hline \multicolumn{5}{|l|}{ LED test result: } \\
\hline On cervical swab, $>$ trace & $87 \cdot 1 \%$ & $80 \cdot 6 \%$ & $1 \cdot 6$ & NS \\
\hline On urine, $>$ trace & $33 \cdot 3 \%$ & $33 \cdot 7 \%$ & $1 \cdot 0$ & NS \\
\hline
\end{tabular}

$\star$ By Fisher's Exact Test. presence of endocervical mucopus (odds ratio $3.0,95 \%$ confidence interval $0 \cdot 9-9 \cdot 0$, $\mathrm{P}=0.06$ ) and for cervical friability (odds ratio $1 \cdot 9,95 \%$ confidence interval $0 \cdot 9-4 \cdot 5, P=$ $0 \cdot 11$ ).

On the other hand, as shown in table 3, vaginal infections, particularly candidiasis and/or trichomoniasis, were associated with the presence of symptoms. Vaginal discharge and/or itch were associated with candidiasis, and there was a trend towards vaginal discharge being associated with trichomoniasis. Both vaginal discharge and itch were associated with the presence of candidiasis and/or trichomoniasis. Furthermore, a positive LED test on either urine or cervical secretions was strongly associated with the presence of vaginal infection, although many uninfected women also had positive LED tests. Among women without evidence of vaginal infection, there was a trend towards an association between a positive urine LED test and the presence of cervical infection (odds ratio 2.5 ), but there were only 14 women without vaginal infection who had cervical infection, and this association did not reach statistical significance $(95 \%$ confidence interval $0 \cdot 7-8 \cdot 6, \mathrm{P}=$ $0 \cdot 13)$.

The accuracy of the WHO algorithm for identifying cervical infection among women with discharge (without a speculum examination $)^{3}$ was evaluated on the 53 women $(18.5 \%)$ who complained of vaginal discharge. Six of these women $(11 \cdot 1 \%)$ had either gonococcal or chlamydial infection, similar to the prevalence of cervical infection among asymptomatic women. According to the WHO algorithm, risk assessment is considered positive (and treatment provided for gonococcal and chlamydial cervical infection) if two or more of the following factors are present: age less than 21 years, unmarried status, report of more than one sex partner, and report of a new sex partner within the past three months. In this population, the sensitivity of the algorithm was $50 \%(3 / 6)$, the specificity $79 \%$

Table 4 Performance of previously developed algorithms in predicting gonococcal and/or chlamydial cervical infection among antenatal women in Nairobi, Kenya without vaginal infection $(N=286)$

\begin{tabular}{|c|c|c|c|c|}
\hline & Infected & Uninfected & Odds Ratio & $P$ Value \\
\hline \multicolumn{5}{|l|}{ Candidiasis: } \\
\hline Vaginal discharge & $25 \cdot 3 \%$ & $16 \cdot 1 \%$ & $2 \cdot 5$ & $0 \cdot 11$ \\
\hline Vaginal itch & $26 \cdot 7 \%$ & $13.7 \%$ & $2 \cdot 3$ & 0.02 \\
\hline Vaginal discharge and/or itch & $41 \cdot 3 \%$ & $22 \cdot 3 \%$ & 2.5 & 0.002 \\
\hline Dyspareunia & $20.0 \%$ & $18 \cdot 0 \%$ & $\overline{1} \cdot 1$ & NS \\
\hline \multicolumn{5}{|l|}{ Trichomoniasis: } \\
\hline Vaginal discharge & $26 \cdot 3 \%$ & $16 \cdot 6 \%$ & $1 \cdot 8$ & $0 \cdot 13$ \\
\hline Vaginal itch & $21 \cdot 1 \%$ & $16 \cdot 2 \%$ & 1.4 & NS \\
\hline Vaginal discharge and/or itch & $33 \cdot 3 \%$ & $25 \cdot 8 \%$ & 1.4 & NS \\
\hline Dyspareunia & $26 \cdot 3 \%$ & $16 \cdot 6 \%$ & 1.8 & $0 \cdot 13$ \\
\hline \multicolumn{5}{|l|}{ Bacterial vaginosis: } \\
\hline Vaginal discharge & $15 \cdot 3 \%$ & $19 \cdot 4 \%$ & 0.8 & NS \\
\hline Vaginal itch & $18 \cdot 6 \%$ & $16 \cdot 7 \%$ & $1 \cdot 1$ & NS \\
\hline Vaginal discharge and/or itch & $25 \cdot 4 \%$ & $27 \cdot 8 \%$ & 0.9 & NS \\
\hline Dyspareunia & $22 \cdot 0 \%$ & $17 \cdot 6 \%$ & $1 \cdot 3$ & NS \\
\hline \multicolumn{5}{|l|}{ Candidiasis and/or trichomoniasis: } \\
\hline Vaginal discharge & $25 \cdot 2 \%$ & $14 \cdot 0 \%$ & $2 \cdot 1$ & 0.03 \\
\hline Vaginal itch & $23.5 \%$ & $12.9 \%$ & $2 \cdot 1$ & 0.03 \\
\hline Vaginal discharge and/or itch & $37 \cdot 4 \%$ & $20 \cdot 5 \%$ & $2 \cdot 3$ & 0.003 \\
\hline Dyspareunia & $22.6 \%$ & $15 \cdot 8 \%$ & $1 \cdot 6$ & $0 \cdot 19$ \\
\hline \multicolumn{5}{|l|}{$\begin{array}{l}\text { LED test result in association } \\
\text { with any vaginal infection: }\end{array}$} \\
\hline On cervical swab, $>$ trace & $\begin{array}{l}90 \cdot 1 \% \\
(\mathrm{~N}=152)\end{array}$ & $\begin{array}{l}71 \cdot 0 \% \\
(\mathbf{N}=131)\end{array}$ & $3 \cdot 7$ & $<0.001$ \\
\hline On urine, $>$ trace & $\begin{array}{l}44 \cdot 4 \% \\
(\mathrm{~N}=117)\end{array}$ & $\begin{array}{l}20.6 \% \\
(\mathrm{~N}=97)\end{array}$ & $3 \cdot 1$ & $<0.001$ \\
\hline
\end{tabular}

Only 214 women had LED tests performed on urine.

\begin{tabular}{|c|c|c|c|}
\hline & \multicolumn{3}{|c|}{ Zaïre algorithm, 1993* } \\
\hline & $G C / C T+$ & $G C / C T-$ & Totals \\
\hline \multirow{8}{*}{$\begin{array}{l}\text { Scoret } \\
\text { Score- } \\
\text { Totals } \\
\text { Sensitivity: } \\
\text { Specificity: } \\
\text { Positive predictive value: }\end{array}$} & 5 & 46 & 51 \\
\hline & 22 & 141 & 163 \\
\hline & $\stackrel{27}{27}$ & 187 & 214 \\
\hline & $19 \%$ & & $72 \%$ \\
\hline & $75 \%$ & & $74 \%$ \\
\hline & $10 \%$ & & $16 \%$ \\
\hline & \multicolumn{3}{|c|}{ Mwanza "R1sim" algorithm $\ddagger$} \\
\hline & $G C / C T+$ & $G C / C T-$ & Totals \\
\hline Scoret & 4 & 32 & 36 \\
\hline Score - & 27 & 233 & 250 \\
\hline \multirow[t]{2}{*}{ Totals } & 31 & 255 & 286 \\
\hline & Kenya & & $\begin{array}{l}\text { Mwanza } \\
\text { (rural) } t\end{array}$ \\
\hline Sensitivity: & $13 \%$ & & $46 \%$ \\
\hline $\begin{array}{l}\text { Specificity: } \\
\text { Positive predictive value: }\end{array}$ & $87 \%$ & & $84 \%$ \\
\hline
\end{tabular}

*From Reference 5. 
(37/47) and the positive predictive value $12 \%$ (3/13).

Table 4 shows the accuracy in this population of risk assessment algorithms developed in two other African countries for predicting the presence of gonococcal and/or chlamydial cervical infection in pregnant women. In the Zaïrian algorithm, ${ }^{5}$ points are allocated as follows: 5 for unmarried status, 10 for reporting more than one sex partner in the past year, 14 for age under 25, 11 for age 25-34, 1 for reporting vaginal discharge, 1 for reporting lower abdominal pain, 10 for a 1 + LED test, 12 for a $2+$ LED test and 15 for a $3+$ LED test. Those women with scores over 28 are considered to have a cervical infection. We only enquired as to new sex partners in the previous three months, so had to use that parameter as a surrogate for sex partners in the past year. The sensitivity of the risk score in identifying cervical infection in the Zaiian population was $72 \%$, but in our population only $19 \%$. Specificities were about $75 \%$ in both populations and positive predictive values were both low. Several potential risk scores have been proposed from work in Mwanza, Tanzania, but the one that performed best in our population was a simplified risk assessment algorithm designated "R1sim". ${ }^{6}$ In this algorithm, points are allocated as follows: 1 for age less than 25 years, 1 for being unmarried (or in a polygamous marriage), 1 for having ever given birth previously, 1 for the last child having been born over five years previously, and 1 for reporting more than one sex partner in the previous year. Those women with scores greater than or equal to 3 are considered to have a cervical infection. Again, we considered new sex partners in the previous three months instead of the previous year as a risk factor, and as we had not asked systematically about previous children, we did not use a history of previous births as a risk factor, and therefore employed a cut-off of 2 rather than 3. The sensitivity of this risk score in identifying cervical infection in the Tanzanian population was $46 \%$, but in our population only $13 \%$. Again, specificities were high (both over $80 \%$ ) and positive predictive values low.

\section{Discussion}

The prevalences found in this study of vaginal and cervical infection, and of STDs in general, were extremely high for what is often considered a "low risk" population. Although chlamydia EIA tests were not confirmed by a blocking antibody assay, in a recent evaluation of the Syva Micro Trak EIA system for detecting chlamydial infection, only $6 \%$ of EIA positive specimens were not confirmed by blocking assay, and in half of these, organisms were detected by direct fluorescent-antibody analysis, suggesting that they were in fact true positives. ${ }^{12}$ It is unlikely therefore that the prevalence of chlamydial infection was significantly over-estimated. On the other hand, as only one cervical specimen was taken from each woman for gonorrhoea culture, the fre- quency of gonorrhoea in the population may indeed have been under-estimated.

Two approaches, aetiology-based and clinical assessment-based, have traditionally been used by health care providers in managing STDs. Management on the basis of aetiology is the ideal approach, but requires laboratory facilities, which are generally not available in the resource-poor settings found in many developing countries. Furthermore, because of travel costs and for other reasons, patients often do not return for follow-up to obtain their results. The clinical diagnostic approach obviates the need for a laboratory, but studies have shown that even highly experienced clinicians fail to make the correct diagnosis and/or miss concurrent infections in a significant number of cases. In a Kenyan study of men with gonococcal and non-gonococcal genital discharges, $19 \%$ were incorrectly classified on the basis of clinical criteria. ${ }^{13}$ In a South African study of 100 men and 100 women with genital ulcers, ${ }^{14}$ clinicians correctly identified only about one-third of the cases of chancroid or syphilis in men, and about onehalf of cases in women, and less than $10 \%$ of mixed infections. Thus, syndromic approaches to identifying sexually transmitted infections in symptomatic individuals have been developed, and risk assessment, using demographic and behavioural information to predict the likelihood of infection, has been advocated for identifying infection among asymptomatic individuals..$^{5-6}$

Unfortunately, in this study, there were very few demographic or behavioural risk factors identified which were associated with the presence of cervical infection. Most of the antenatal women in the study were married and reportedly monogamous. This is consistent with previous findings from Kenya that it is primarily the behaviour of their male sex partners which puts married women in Kenya at risk of STDs. ${ }^{15}$ Previous studies from Zaïre and Tanzania ${ }^{6}$ have identified the demographic factors of young age (under 25) and being unmarried as important risk factors for cervical infection in antenatal women, and these two factors have been key ones in driving the development of risk scores for predicting the presence of cervical infection. As these two factors were not associated with cervical infection in our population, it is not surprising that the scoring systems developed in Zaire and Tanzania did not perform well. The presence of vaginal discharge and a positive LED test, which were predictive of cervical infection in Zaïre (and to a lesser extent in Tanzania) were also not associated with cervical infection in our population, but rather were associated with vaginal infection, which was extremely common. It is thus likely that the presence of vaginal infection overwhelmed any effect of cervical infection on the LED test. The best predictors of cervical infection in our population were the presence of cervical friability or endocervical mucopus, similar to previous findings reported from Kenya. ${ }^{4}$ Most health practitioners would likely diagnose cervical infection on the basis of these signs, but 
they are only useful in situations where speculum examinations can be performed. This unfortunately is not the case in most primary care settings in sub-Saharan Africa.

Treating all women with vaginal discharge for cervical infection, as is recommended in some syndromic approaches, would in this population have entailed treating $19 \%$ of the population, and would still have missed $81 \%$ of all cervical infections. Using the combination of symptoms and risk assessment recommended in the current WHO guidelines for women presenting to a health provider with vaginal discharge would have entailed treating about $5 \%$ of the population, but would have missed $90 \%$ of all cervical infections.

The detection and management of cervical infection in women, whether they have symptoms or not, is a difficult challenge. Further work on risk assessment and symptom-based approaches to case detection among antenatal women is required before guidelines can be generalised. As the behaviour of male partners may be an important factor predictive of risk for STDs in some populations of women, measures of perceived male risk behaviour should be evaluated in this respect and perhaps incorporated into risk scoring systems. It may also turn out that different risk scores will be required in different geographical locations, and that local research will therefore have to be conducted before specific recommendations can be made. If this is so, then the use of risk assessment for antenatal women may prove to be impracticable. However, syndromic approaches and risk assessment may have more validity in other accessible populations of women, such as family planning clinic attenders and women who present to health facilities specifically because of genital tract symptoms such as vaginal discharge, dysuria or lower abdominal pain. More research on STD case detection in such populations, including the use of syndromic approaches and risk assessment, is urgently required. As has been observed in the past, ${ }^{16}$ the burden of STDs and reproductive tract infections among African women is great, and little progress has been made in reducing it.

1 Moses S, Ngugi EN, Bradley JE, Njeru EK, Eldridge G, Muia E, Olenja J, Plummer FA. Health care-seeking behavior related to the transmission of sexually transmitted diseases in Kenya. Am $\mathcal{F}$ Public Health 1994;84 1947-51.

2 Lande R. Controlling sexually transmitted diseases. Popul Rep, Series L, No. 9. Baltimore: Johns Hopkins School of Public Health, Population Information Program, June 1993.

3 World Health Organization. Recommendations for the Management of Sexually Transmitted Diseases. Advisory group meeting on sexually transmitted diseases treatments, February 18-19, 1993. Geneva: World Health Organization, 1993 .

4 Braddick MR, Ndinya-Achola JO, Mirza NB, Plummer Braddick MR, Ndinya-Achola JO, Mirza NB, Plummer
FA, Irungi G, Sinei SKA, et al. Towards developing a diagnostic algorithm for Chlamydia trachomatis and Neisseria gonorrhoeae cervicitis in pregnancy. Genitourin Med 1990;66:62-5.

5 Vuylsteke B, Laga M, Alary M, et al. Clinical algorithms for the screening of women for gonococcal and chlamydial infection: evaluation of pregnant women and prostitutes in Zaïre. Clin Infect Dis 1993;17:82-8.

6 Mayaud P, Grosskurth H, Changalucha J, et al. Risk assessment and other screening options for gonorrhoea and chlamydial infections in women attending rural Tanzanian antenatal clinics. Bull WHO, 1995;73:621-30.

7 Mayaud P, Changalucha J, Grosskurth $\mathrm{H}$, et al. The value of urine specimens in screening for male urethritis and its urine specimens in screening for male urethritis and its
microbial aetiologies in Tanzania. Genitourin Med 1992; microbial

8 Sellors JW, Mahony JB, Pickard L, et al. Screening urine with a leukocyte esterase strip and subsequent chlamydial testing of asymptomatic men attending primary care practitioners. Sex Transm Dis 1993;20:152-7.

9 Johnson J, Neas B, Parker D, Fortenberry JD, Cowan LD. Screening for urethral infection in adolescent and youn adult males. F Adolesc Health 1993;14:356-61.

10 Knud-Hansen CR, Dallabetta GA, Reichart C, Pabst KM Hook EW, Wasserheit JN. Surrogate methods to diagnose gonococcal and chlamydial cervicitis: comparison of leukocyte esterase dipstick, endocervical gram stain and culture. Sex Transm Dis 1991;18:211-6.

11 Hillier $\mathrm{H}$, Holmes $\mathrm{KK}$. Bacterial vaginosis. In: Holme KK, Mårdh P-A, Sparling PF, Wiesner PJ, eds. Sexually Transmitted Diseases. New York: McGraw-Hill, 1990 547-59.

12 Nachamkin I, Riddle DL, O'Connor J. Blocking antibody assay for confirmation of urogenital Chlamydia infection. Clinical \& Diagnostic Laboratory Immunology 1995;2 634-5.

13 Ndinya-Achola JO, Kihara AN, Fisher LD, Krone MR Plummer FA, Holmes KK. Clinical etiologic diagnosis of genital discharge in males [Abstract 179B]. In Abstract Book, Volume I, X International Conference on AIDS Yokohama, Japan, 7-12 August 1994

14 O'Farrell N, Hoosen AA, Coetzee KD, van den Ende J. Accuracy of clinical diagnosis in genital ulcer disease in Accuracy of clinical diagnosis in genital ulcer disease in Durban, South Africa [Abstract PO-C10-2819]. In
Abstract Book, Volume II, IX International Conference on AIDS/IV STD World Congress, Berlin, Germany, 6-1 June, 1993.

15 Moses S, Muia E, Bradley JE, et al. Sexual behaviour in Kenya: implications for sexually transmitted disease transmission and control. Soc Sci Med 1994;39:1649-56.

16 DeCosas J. Time to stop culturing silence. Commentary. Lancet $1995 ; 345 \cdot 270-1$ 